Research Article

\title{
Elastic Properties of Liquid Surfaces Coated with Colloidal Particles
}

\author{
Edward Bormashenko, ${ }^{1,2}$ Gene Whyman, ${ }^{1}$ and Oleg Gendelman ${ }^{3}$ \\ ${ }^{1}$ Physics Department, Ariel University, P.O. Box 3, 40700 Ariel, Israel \\ ${ }^{2}$ Chemical Engineering and Biotechnology Department, Ariel University, P.O. Box 3, 40700 Ariel, Israel \\ ${ }^{3}$ Faculty of Mechanical Engineering, Technion-Israel Institute of Technology, 32000 Haifa, Israel
}

Correspondence should be addressed to Edward Bormashenko; edward@ariel.ac.il

Received 23 March 2015; Accepted 24 May 2015

Academic Editor: Mohindar S. Seehra

Copyright (C) 2015 Edward Bormashenko et al. This is an open access article distributed under the Creative Commons Attribution License, which permits unrestricted use, distribution, and reproduction in any medium, provided the original work is properly cited.

\begin{abstract}
The physical mechanism of elasticity of liquid surfaces coated with colloidal particles is proposed. It is suggested that particles are separated by water clearings and the capillary interaction between them is negligible. The case is treated when the colloidal layer is deformed normally to its surface. The elasticity arises as an interfacial effect. The effective Young modulus of a surface depends on the interfacial tension, equilibrium contact angle, radius of colloidal particles, and their surface density. For the nanometrically scaled particles the line tension becomes essential and has an influence on the effective Young modulus.
\end{abstract}

\section{Introduction}

Colloidal particles attached to liquid surfaces are abundant in many products and processes, including crude-oil emulsions, food foams, and flotation and were studied intensively in the last decade $[1,2]$. When colloidal particles are attached to liquid/liquid interfaces they can work as surfactants, promoting stabilization of emulsions [3, 4]. Colloidal particles attached to liquid surface gave rise to colloidosomes, that is, selectively permeable capsules demonstrating a potential for drug delivery [5]. When attached to liquid droplets' surfaces, colloidal particles allowed manufacturing the so-called liquid marbles, which are nonstick droplets, presenting an alternative to the lotus-inspired superhydrophobicity [6-13]. Liquid marbles demonstrated a diversity of promising applications, including encapsulation, microfluidics, cultivation of microorganisms, gas-sensing, miniaturized synthesis, and water storing [1420].

Solid particles attached to liquid surface initiate a broad diversity of physical effects including capillarity-induced selfassembly and particle-assisted wetting [21-23]. One of the most interesting phenomena related to the ensembles of colloidal particles located at the liquid/air interfaces is the quasielastic behavior of such systems [24-26]. Vella et al. proposed the physical mechanism of elasticity demonstrated by the layer of colloidal particles covering a liquid surface. In our paper we introduce an alternative mechanism of elasticity of these layers.

\section{Results and Discussion}

It was demonstrated recently that liquid surfaces coated with colloidal particles behave as two-dimensional elastic solids (and not liquids) when deformed [24-26]. For example, liquid marbles do not coalesce when pressed one to another, as shown in Figure 1. When liquid marbles collide they do not coalesce but demonstrate quasielastic (almost elastic, noncoalescent) collision depicted in Figure 2. It was also demonstrated that the layers built of monodisperse colloidal particles can support anisotropic stresses and strains [26]. The observed pseudoelastic properties of liquid surfaces coated with colloidal particles call for explanation.

Vella et al. treated the collective behavior of a closepacked monolayer of non-Brownian colloidal particles placed at a fluid-liquid interface [26]. In this simplest case, however, the close-packed monolayers may be characterized using effective Young's modulus and Poisson ratio [26]. These 


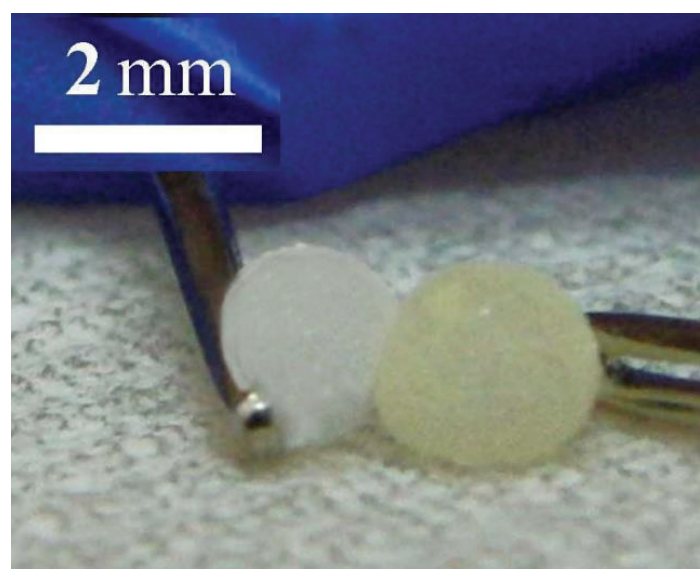

Figure 1: $10 \mu \mathrm{L}$ Teflon (white) and lycopodium (yellow) coated marbles do not coalesce even when pressed one to another.

authors proposed an expression for the effective Young modulus $E$ of the "interfacial particle raft" in the form [26]

$$
E \cong \frac{1-\nu}{1+\phi} \frac{\gamma}{d}
$$

where $\gamma$ is the surface tension of liquid, $d$ is the diameter of solid particles, $\nu$ is the Poisson ratio of solid particles, and $\phi$ is the solid fraction of the interface. They concluded that the elastic properties of such an interface are not dependent on the details of capillary interaction between particles [26].

The model presented by Vella et al. implies close packing of elastic spheres, coating liquid marbles. However, in the case of liquid marbles, for example, the surface is not completely coated by solid particles, as shown in Figures 3(a)-3(b), representing ESEM images of liquid marbles coated with lycopodium (for the detailed discussion of wetting of lycopodium particles see [27]) and with polyvinylidene fluoride (PVDF) particles. Water clearings separating particles and aggregates of particles are distinctly seen [28]. It should also be emphasized that liquid marbles demonstrate "transversal" (normal to their surface) and not lateral pseudoelasticity when being pressed or collide, whereas the model developed in [26] treats only the lateral elasticity of surfaces, coated with solid particles.

We propose an alternative mechanism of pseudoelasticity of liquid surface covered by solid particles, which are not close-packed, explaining the "transversal" elasticity of surfaces, depicted in Figure 4. As it will be seen below, the elasticity in this case is caused by the change in the liquid area under deformation, as it also occurs in [26].

Consider two media (one of which may be vapor) in contact (Figure 5). The plane boundary between them is characterized by the specific surface energy (or the surface tension) $\gamma_{1,2}$. At this surface floats a spherical body of the radius $R$ modeling a colloidal particle. Note that this flotation is due to surface forces but not to gravity since the size of a colloidal particle is much lower than the capillarity length $\sqrt{\gamma_{1,2} / g\left|\rho_{1}-\rho_{2}\right|}$ ( $\rho_{1}$ and $\rho_{2}$ are the corresponding densities). Beside $\gamma_{1,2}$, these forces are characterized by the surface tensions $\gamma_{1}$ and $\gamma_{2}$ at the corresponding solid-fluid interfaces (see Figure 5). The capillary length describes the competition between surface tension and gravity (including buoyancy). For objects' lengths substantially lower than the capillary length, the gravity effects are negligible when compared to surface tension.

The total surface energy $U$ is given by

$$
\begin{aligned}
U= & 2 \pi R(R-h) \gamma_{1}+2 \pi R(R+h) \gamma_{2}-\pi \gamma_{1,2}\left(R^{2}-h^{2}\right) \\
& +2 \pi \Gamma \sqrt{R^{2}-h^{2}} .
\end{aligned}
$$

The first and second terms represent the surface energies of the solid-fluid interfaces, while the third one describes the energy of the "disappearing" area due to the solid body. Also the so-called line tension, $\Gamma$, of the triple line, neighboring two fluid and one solid media, is included in (2) since it may be important for very small particles $[29,30]$. As is known, the flotation of strongly hydrophilic nanoparticles may be explained by considering the line tension only.

The equilibrium depth of immersion $h_{0}$ can be found by differentiation of (2):

$$
\frac{\gamma_{2}-\gamma_{1}}{\gamma_{1,2}}+\frac{h_{0}}{R}-\frac{\Gamma}{\gamma_{1,2} R} \cdot \frac{h_{0}}{\left(R^{2}-h_{0}^{2}\right)^{1 / 2}}=0 .
$$

In the widespread case when $\Gamma \ll \gamma_{1,2} R$, one can write down the approximate explicit solution of (3), as it was done in [29]. For this purpose, $h_{0}$ and the square root in (3) are expended in powers of the dimensionless parameter $\kappa$ defined as

$$
\kappa=\frac{\Gamma}{\gamma_{1,2} R}
$$

and the result for equilibrium depth of floating is

$$
h_{0}=R(\cos \theta+\kappa \cot \theta) \text {. }
$$

Here we also use notation for the Young contact angle

$$
\cos \theta=\frac{\gamma_{1}-\gamma_{2}}{\gamma_{1,2}}
$$

To provide a correct asymptotic behavior of expression of $h_{0}$ in powers of $\kappa$ one should require $\kappa / \sin \theta \ll 1$ since the coefficients in the above-mentioned expansion may increase for the lowest and highest contact angles $\theta$. In order to study the oscillatory response of the surface covered by the colloidal particles, we obtain for the force component acting on the solid body in the case of small deviations from the equilibrium depth $h_{0}$

$$
F_{h}=-\frac{d U}{d h}=-2 \pi \gamma_{1,2}\left(1-\frac{\kappa}{\sin ^{3} \theta}\right) \cdot\left(h-h_{0}\right)
$$

which is of a Hooke law form.

As it follows from (7), our model system is a harmonic oscillator with the eigenfrequency

$$
\omega=\sqrt{\frac{2 \pi \gamma_{1,2}\left(1-\kappa / \sin ^{3} \theta\right)}{m}}
$$




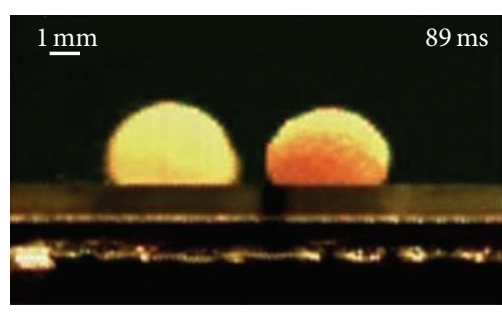

(a)

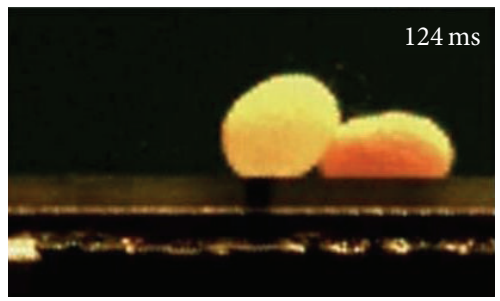

(d)

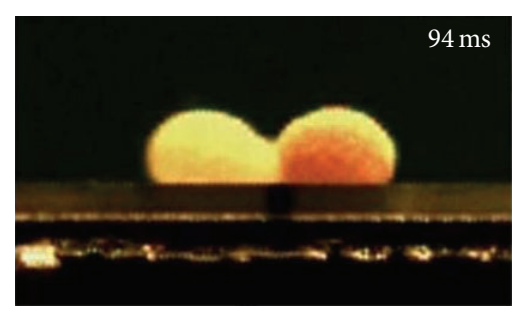

(b)

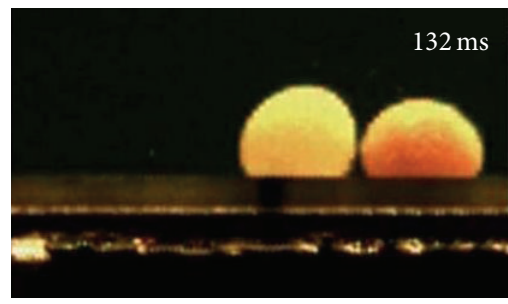

(e)

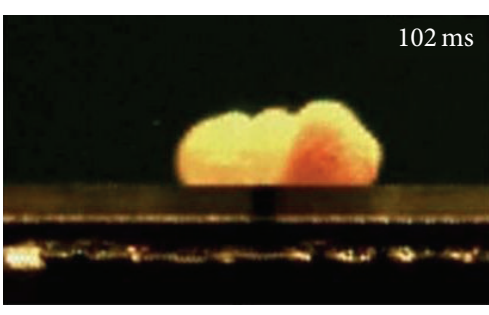

(c)

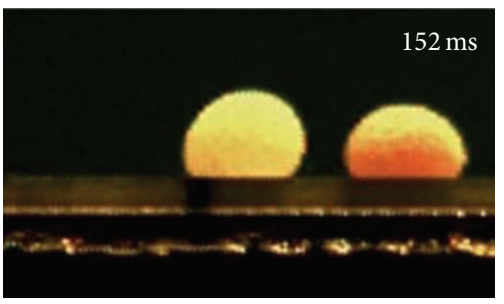

(f)

FIGURE 2: Sequence of images illustrating the quasielastic collision between lycopodium coated liquid marbles. Scale bar is $1 \mathrm{~mm}$.

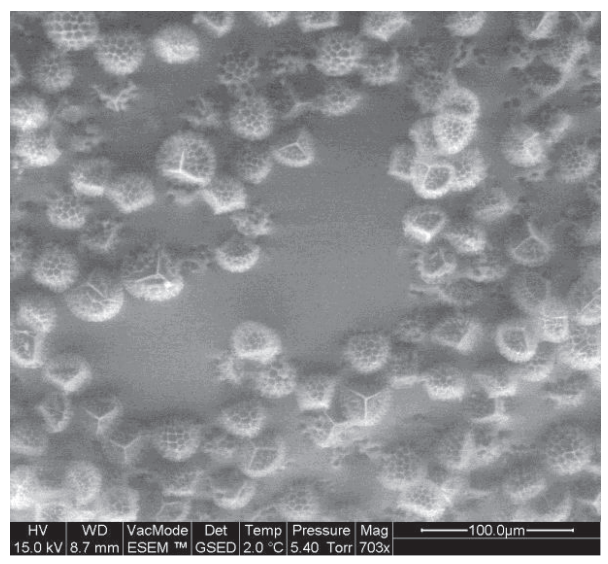

(a)

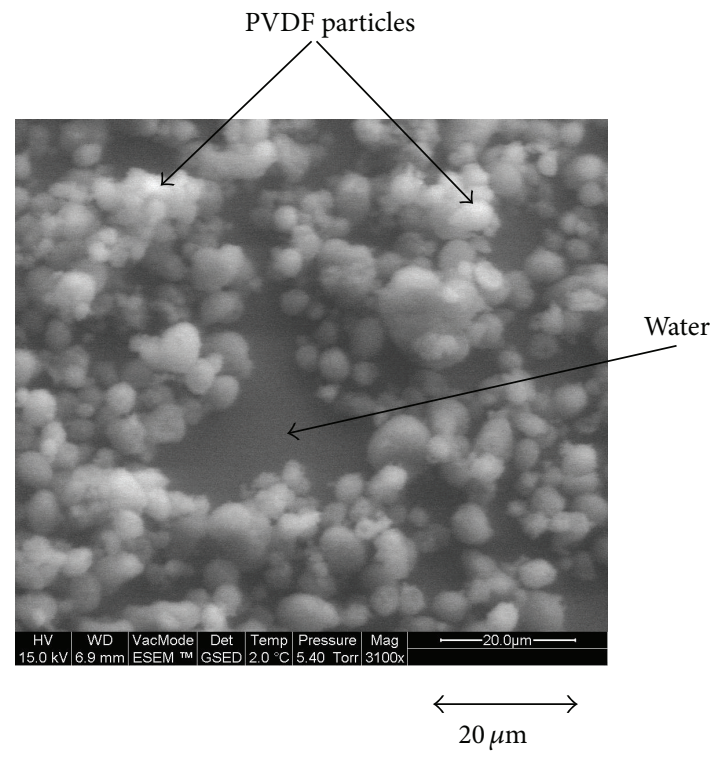

(b)

FIGURE 3: (a) ESEM image of the surface of liquid marble coated with lycopodium particles. Scale bar is $100 \mu \mathrm{m}$. (b) ESEM image of the marble's surface (white spots are aggregates comprising particles of PVDF). Scale bar is $20 \mu \mathrm{m}$.

( $m$ is the mass of the floating body; (8) is obviously applicable when $\left.\kappa / \sin ^{3} \theta<1\right)$. Note that elastic properties do not depend on the surface tensions $\gamma_{1}$ and $\gamma_{2}$ which determine only the equilibrium of the system in (5), (6). Equation (8) may be rewritten in the form revealing an explicit dependence of the eigenfrequency on the radius of a particle $R$ :

$$
\omega=l_{\text {ca }} \sqrt{\frac{3}{2} \frac{g}{R^{3}}\left(1-\frac{\kappa}{\sin ^{3} \theta}\right)},
$$

where $l_{\mathrm{ca}}=\sqrt{\gamma_{1,2} / \rho_{p} g}$ with $\rho_{p}$ being the density of a colloidal particle.
Now let $n$ be the surface density of colloidal particles on the area $S$ of the boundary (the surface is supposed to be flat). Under the deviation $h-h_{0}$, the total elastic force on the area $S$ is $n S F_{h}$. According to the Hooke law

$$
\frac{n S F_{h}}{S}=E \frac{h-h_{0}}{\left|h_{0}\right|}
$$

and for the effective Young modulus $E$ it follows on account of (7)

$$
\begin{aligned}
E & =2 \pi n\left|h_{0}\right| \gamma_{1,2}\left(1-\frac{\kappa}{\sin ^{3} \theta}\right) \\
& \approx 2 \pi n R \gamma_{1,2}\left|\cos \theta-\kappa \cot ^{3} \theta\right| .
\end{aligned}
$$




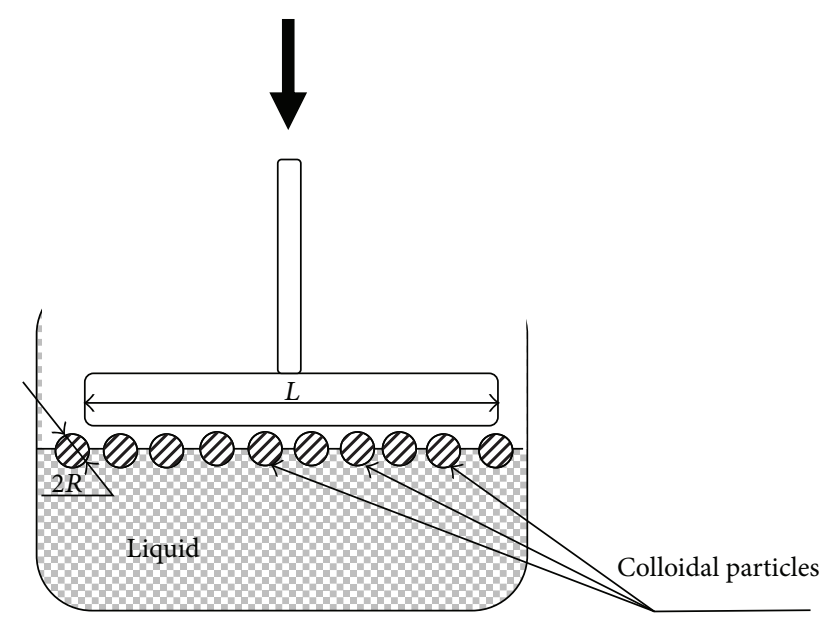

FIGURE 4: The transversal elasticity of a liquid surface, coated by the colloidal layer: the layer is pressed by a plate moving normally to the surface.

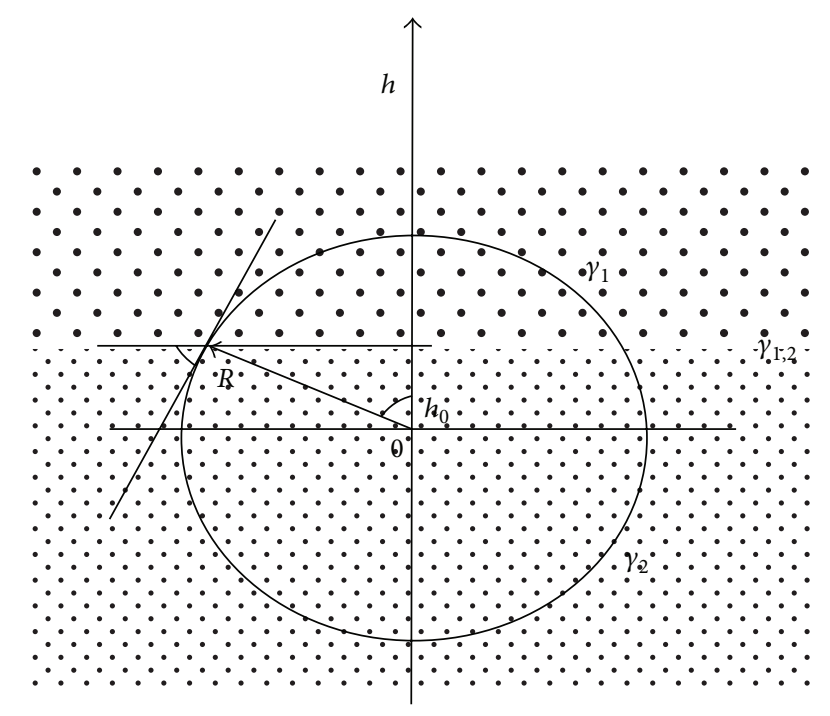

Figure 5: Colloidal particle with the radius, $R$, located at the interface separating two media.

Note that here the deformation is connected with the interface change but not with the deformation of particles. The whole approach is applicable for dilute colloids or colloids with a weak interparticle interaction. This assumption is justified for colloidal particles with the characteristic dimensions of particles $R \leq 5 \mu \mathrm{m}$, as shown in [21]. Such particles do not deform the water/vapor interface; this leads to a negligible capillary interaction [21]. Equation (10) will predict the effective elastic modulus for both of flat and curved surfaces coated by colloidal particles, in the case when the characteristic dimension of the deformed area $L$ is much larger than that of a particle $R$ (see Figure 4 ). It should be stressed that (10) supplies the upper limit of the effective elastic modulus, because it assumes the simultaneous contact of a plate with all of colloidal particles.

An example of numerical estimation of (10) may be given using Figure 3(a). The radius of particles is of order $R \sim 1 \mu \mathrm{m}$; thus, taking into account the most reasonable estimation of line tension $\Gamma \sim 10^{-10} \mathrm{~N}$ [30] and water surface tension $\gamma_{1,2} \sim 10^{-1} \mathrm{~N} / \mathrm{m}$, we see that according to $(4) \kappa \sim 10^{-3}$ and line tension is negligible. From Figure $3(\mathrm{a}), n \sim 10^{9} \mathrm{~m}^{-2}$, for lycopodium $\theta \sim 120^{\circ}$, and from (10) a realistic estimation follows $E \sim 100 \mathrm{~Pa}$ that is two orders of magnitude lower than that following from (1) in the case of close-packed microparticles. Recall that our model implies a "dilute" distribution of particles on the surface of liquid, presuming nondirect contact situation, in which solid particles are separated by water clearings, as it occurs on the surfaces of liquid marbles (see Figures 3(a)-3(b)). The values of the effective elastic modulus, which were recently established experimentally in [31] under deformation of liquid marbles coated by micrometrically scaled polyethylene particles are close to $100 \mathrm{~Pa}$, in good agreement with our estimation.

For nanometric particles, the line tension should be taken into account in (8) and (10). For example, for hydrophobic colloidal particles of a size $R=10 \mathrm{~nm}$ on water/air interface with above-mentioned parameters we have $\kappa \sim 0.1$ and putting the average distance between particles equal to their size $R$, the concentration $n \sim 10^{16} \mathrm{~m}^{-2}$. Then from (10) it follows $E \sim 10 \mathrm{MPa}$ that is much more than that in the case of microscaled particles. This is not surprising; (1) yields for nanoparticles similar values of the elastic modulus. It is also obvious from (10) that, in the case of materials with lower values of the contact angle $\theta$, the value of the effective Young modulus will be much lower.

It is noteworthy that the proposed model of transversal elasticity of surfaces coated with colloidal particles implies zero contact angle hysteresis [32-34]. Considering the contact angle hysteresis will call for much more complicated mathematical treatment.

It should be stressed that the proposed mechanism of elasticity will work only at the initial stage of the deformation of droplets coated with colloidal particles (see Figures 1-2), on the "short" time spans of deformation $\tau_{1} \cong R / v$, where $v$ is the characteristic velocity of the deformation; at the characteristic times, given by $\tau_{2} \cong a / v$, where $a$ is the characteristic dimension of an entire droplet, the mechanisms of elasticity discussed in [31, 35] will be dominant (obviously $\tau_{1} \ll \tau_{2}$ takes place). The characteristic time of deformation of water droplets bouncing solid substrates was established in [35] as $\tau_{2} \cong \sqrt{\rho a^{3} / \gamma}$. This is not surprising, because in the case of bouncing droplets the role of the effective spring stiffness $k$ is played by the surface tension of a bouncing drop. Thus, the effective mechanical scheme of a liquid surface coated with solid colloidal particles looks like the case that is depicted in Figure 6 ( $\eta$ is the viscosity of the liquid). The characteristic time of the processes driven by viscosity $\tau_{3}$ is given by $\tau_{3} \cong$ $a \eta / \gamma$. Thus, the time scaling of the deformation is determined by the hierarchy of the triad of the time scales: $\tau_{1}, \tau_{2}$, and $\tau_{3}$.

\section{Conclusions}

We introduce the mechanisms of elasticity of liquid surfaces coated with colloidal particles in the case when the gravity and capillary interactions are negligible. We treat the situation 


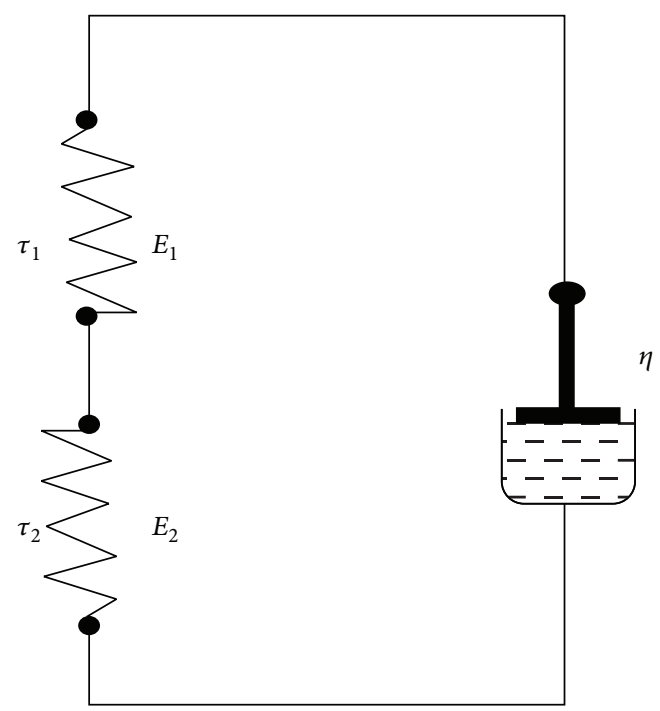

FIGURE 6: Effective mechanical scheme of a liquid surface coated with colloidal particles $\left(E_{1}, E_{2}\right.$ are the effective Young moduli; $\eta$ is the viscosity of the liquid).

when solid colloidal particles do not form close packaging and are separated by water clearings. The deformation normal to the surface of a colloidal layer is discussed. When a colloidal particle is displaced from its equilibrium position the pseudoelastic force following the Hooke-like law arises. This purely interfacial force gives rise to the pseudoelasticity of the colloidal raft coating a liquid surface.

The effective Young modulus of a surface depends on the interfacial tension, equilibrium contact angle, radius of colloidal particles, and their surface density. Eigenfrequency of oscillations of colloidal particles is calculated. For the nanometrically scaled particles the line tension may dominate over interfacial tension effects. The proposed mechanism of elasticity will work only at the initial stage of the deformation, on "short" time spans of deformation.

\section{Conflict of Interests}

The authors declare that there is no conflict of interests regarding the publication of this paper.

\section{Acknowledgment}

Acknowledgment is made to the donors of the American Chemical Society Petroleum Research Fund for support of this research (Grant no. 52043-UR5).

\section{References}

[1] B. P. Binks and T. S. Horozov, Colloidal Particles at Liquid Interfaces, Cambridge University Press, Cambridge, UK, 2006.

[2] B. P. Binks and R. Murakami, "Phase inversion of particlestabilized materials from foams to dry water," Nature Materials, vol. 5, no. 11, pp. 865-869, 2006.
[3] B. P. Binks, "Particles as surfactants-similarities and differences," Current Opinion in Colloid \& Interface Science, vol. 7, no. 1-2, pp. 21-41, 2002.

[4] R. Aveyard, B. P. Binks, and J. H. Clint, "Emulsions stabilised solely by colloidal particles," Advances in Colloid \& Interface Science, vol. 100-102, pp. 503-546, 2003.

[5] A. D. Dinsmore, M. F. Hsu, M. G. Nikolaides, M. Marquez, A. R. Bausch, and D. A. Weitz, "Colloidosomes: selectively permeable capsules composed of colloidal particles," Science, vol. 298, no. 5595, pp. 1006-1009, 2002.

[6] P. Aussillous and D. Quéré, "Liquid marbles," Nature, vol. 411, no. 6840, pp. 924-927, 2001.

[7] J. Tian, T. Arbatan, X. Li, and W. Shen, "Liquid marble for gas sensing," Chemical Communications, vol. 46, no. 26, pp. 47344736, 2010.

[8] D. Dupin, S. P. Armes, and S. Fujii, "Stimulus-responsive liquid marbles," Journal of the American Chemical Society, vol. 131, no. 15, pp. 5386-5387, 2009.

[9] D. Zang, Z. Chen, Y. Zhang, K. Lin, X. Geng, and B. P. Binks, "Effect of particle hydrophobicity on the properties of liquid water marbles," Soft Matter, vol. 9, no. 20, pp. 5067-5073, 2013.

[10] M. Dandan and H. Y. Erbil, "Evaporation rate of graphite liquid marbles: comparison with water droplets," Langmuir, vol. 25, no. 14, pp. 8362-8367, 2009.

[11] E. Bormashenko, Y. Bormashenko, R. Pogreb, and O. Gendelman, "Janus droplets: liquid marbles coated with dielectric/semiconductor particles," Langmuir, vol. 27, no. 1, pp. 7-10, 2011.

[12] E. Bormashenko, "Liquid marbles: properties and applications," Current Opinion in Colloid and Interface Science, vol. 16, no. 4, pp. 266-271, 2011.

[13] E. Bormashenko, "New insights into liquid marbles," Soft Matter, vol. 8, no. 43, pp. 11018-11021, 2012.

[14] A. V. Rao, M. M. Kulkarni, and S. D. Bhagat, "Transport of liquids using superhydrophobic aerogels," Journal of Colloid and Interface Science, vol. 285, no. 1, pp. 413-418, 2005.

[15] E. Bormashenko, R. Pogreb, Y. Bormashenko, A. Musin, and T. Stein, "New investigations on ferrofluidics: ferrofluidic marbles and magnetic-field-driven drops on superhydrophobic surfaces," Langmuir, vol. 24, no. 21, pp. 12119-12122, 2008.

[16] Y. Zhao, Z. Xu, M. Parhizkar, J. Fang, X. Wang, and T. Lin, "Magnetic liquid marbles, their manipulation and application in optical probing," Microfluidics and Nanofluidics, vol. 13, no. 4, pp. 555-564, 2012.

[17] J. Tian, N. Fu, X. D. Chen, and W. Shen, "Respirable liquid marble for the cultivation of microorganisms," Colloids and Surfaces B: Biointerfaces, vol. 106, pp. 187-190, 2013.

[18] D. Matsukuma, H. Watanabe, M. Minn et al., "Preparation of poly(lactic-acid)-particle stabilized liquid marble and the improvement of its stability by uniform shell formation through solvent vapor exposure," RSC Advances, vol. 3, no. 21, pp. 78627866, 2013.

[19] L. Forny, I. Pezron, K. Saleh, P. Guigon, and L. Komunjer, "Storing water in powder form by self-assembling hydrophobic silica nanoparticles," Powder Technology, vol. 171, no. 1, pp. 1524, 2007.

[20] Y. Chu, Z. Wang, and Q. Pan, "Constructing robust liquid marbles for miniaturized synthesis of graphene/ag nanocomposite," ACS Applied Materials \& Interfaces, vol. 6, no. 11, pp. 8378-8386, 2014. 
[21] P. A. Kralchevsky and K. Nagayama, "Capillary interactions between particles bound to interfaces, liquid films and biomembranes," Advances in Colloid \& Interface Science, vol. 85, no. 2, pp. 145-192, 2000.

[22] A. E. Larsen and D. G. Grier, "Like-charge attractions in metastable colloidal crystallites," Nature, vol. 385, no. 6613, pp. 230-233, 1997.

[23] H. Xu and W. A. Goedel, "Particle-assisted wetting," Langmuir, vol. 19, no. 12, pp. 4950-4952, 2003.

[24] C. Planchette, A.-L. Biance, O. Pitois, and E. Lorenceau, "Coalescence of armored interface under impact," Physics of Fluids, vol. 25, no. 4, Article ID 042104, 2013.

[25] C. Planchette, E. Lorenceau, and A.-L. Biance, "Surface wave on a particle raft," Soft Matter, vol. 8, no. 8, pp. 2444-2451, 2012.

[26] D. Vella, P. Aussillous, and L. Mahadevan, "Elasticity of an interfacial particle raft," Europhysics Letters, vol. 68, no. 2, pp. 212-218, 2004.

[27] E. Bormashenko, T. Stein, R. Pogreb, and D. Aurbach, “Petal effect' on surfaces based on lycopodium: high-stick surfaces demonstrating high apparent contact angles," Journal of Physical Chemistry C, vol. 113, no. 14, pp. 5568-5572, 2009.

[28] E. Bormashenko, Y. Bormashenko, A. Musin, and Z. Barkay, "On the mechanism of floating and sliding of liquid marbles," ChemPhysChem, vol. 10, no. 4, pp. 654-656, 2009.

[29] E. Bormashenko and G. Whyman, "On the role of the line tension in the stability of cassie wetting," Langmuir, vol. 29, no. 18, pp. 5515-5519, 2013.

[30] T. Pompe, A. Fery, and S. Herminghaus, "Measurement of contact line tension by analysis of the three-phase boundary with nanometer resolution," in Apparent and Microscopic Contact Angles, J. Drelich, J. S. Laskowski, and K. L. Mittal, Eds., pp. 3-12, VSP, Utrecht, The Netherlands, 2000.

[31] S. Asare-Asher, J. N. Connor, and R. Sedev, "Elasticity of liquid marbles," Journal of Colloid and Interface Science, vol. 449, pp. 341-346, 2015.

[32] R. Tadmor, "Line energy and the relation between advancing, receding, and Young contact angles," Langmuir, vol. 20, no. 18, pp. 7659-7664, 2004.

[33] R. Tadmor, P. Bahadur, A. Leh, H. E. N'Guessan, R. Jaini, and L. Dang, "Measurement of lateral adhesion forces at the interface between a liquid drop and a substrate," Physical Review Letters, vol. 103, no. 26, Article ID 266101, 2009.

[34] E. Bormashenko, Wetting of Real Surfaces, De Gruyter, Berlin, Germany, 2013.

[35] D. Richard, C. Clanet, and D. Quéré, "Contact time of a bouncing drop," Nature, vol. 417, no. 6891, p. 811, 2002. 

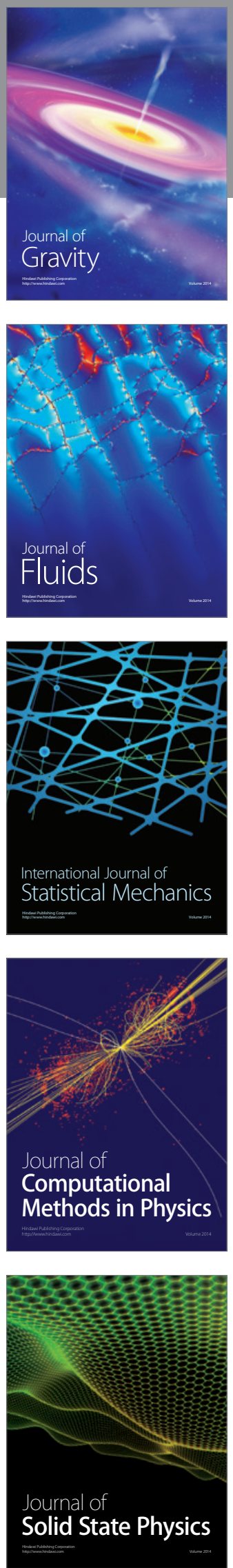

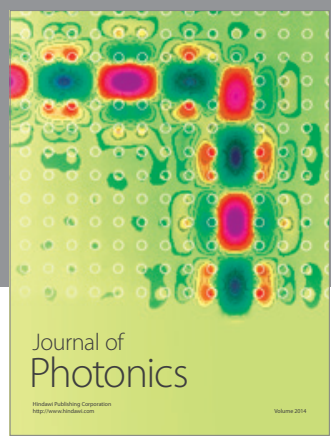

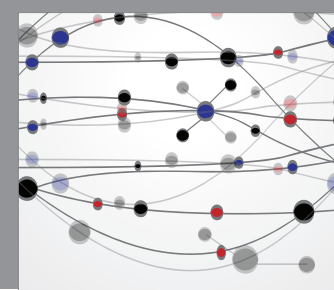

The Scientific World Journal

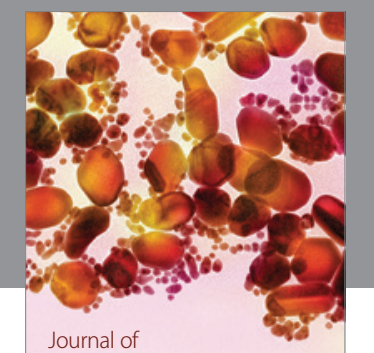

Soft Matter
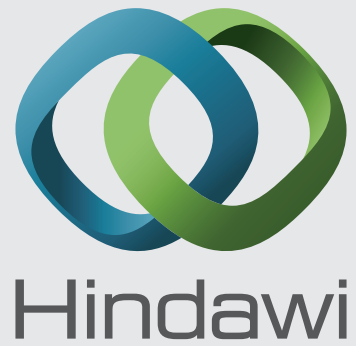

Submit your manuscripts at

http://www.hindawi.com
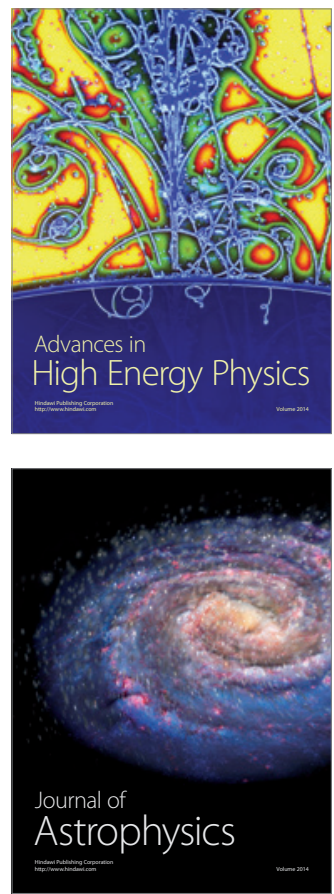
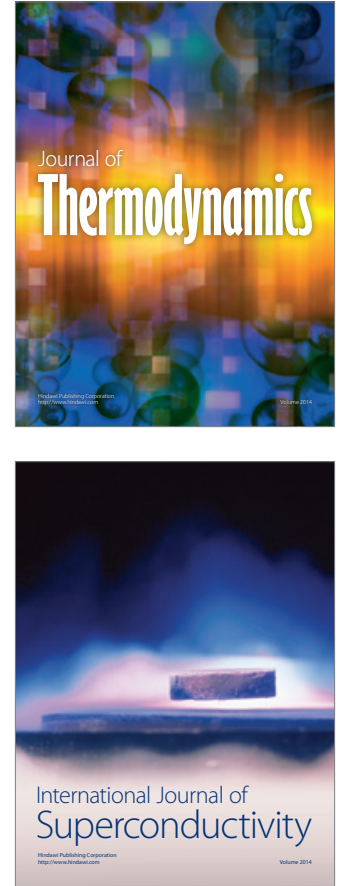
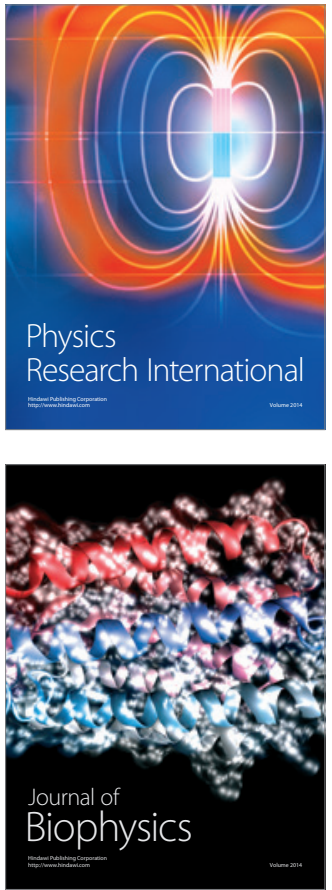
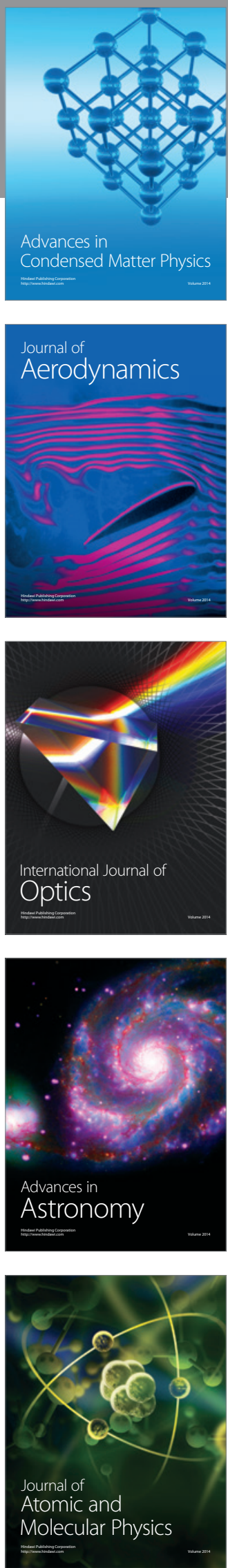